\title{
BP-OP-1-7
}

\section{MCT4 as a potential therapeutic target to augment gemcitabine chemosensitivity in resected pancreatic cancer}

\author{
Sung Hwan LEE ${ }^{1,2}$, Ho Kyoung HWANG ${ }^{3,4}$, Woo Jung LEE ${ }^{3,4}$, Chang Moo KANG ${ }^{* 3,4}$ \\ 'Department of Surgery, CHA Bundang Medical Center, CHA University School of Medicine, Seongnam, Korea \\ ${ }^{2}$ Laboratory of HBP Integrative Precision Oncology, CHA Bio Complex, CHA Health System, Seongnam, Korea \\ ${ }^{3}$ Division of Hepatobiliary and Pancreatic Surgery, Department of Surgery, Yonsei University College of Medicine, Seoul, Korea \\ ${ }^{4}$ Pancreatobiliary Cancer Center, Yonsei Cancer Center, Severance Hospital, Seoul, Korea
}

Introduction: Pancreatic cancer is a devastating disease with a high relapse rate, even in resectable pancreatic cancer. This study aimed to identify the prognostic significance and therapeutic chance of the metabolic subtypes for resectable pancreatic cancer.

Methods: We obtained transcriptomic data from the TCGA-PAAD cohort via the The Cancer Genome Atlas (TCGA) data portal $(n=182)$. After integrative analysis of transcriptomic data in the discovery cohort, immunohistochemical (IHC) staining was performed in the independent cohort to validate the molecule of interest. Experimental testing for the molecule of interest was performed using pancreatic cancer cell lines, including AsPC1, BxPC3, MIA PaCa-2, and PANC-1 in vitro.

Results: Two subtypes showing distinct gene expression patterns in the TCGA-PAAD dataset were identified. The active glucose metabolism subtype showed significantly lower survival regarding cancer relapse after surgical resection. The genes SLC2A1 (GLUT1) and SLC16A3 (MCT4) were highly enriched in the active metabolism subtype. The validation cohort showed high IHC staining intensity for MCT4 and a significantly high relapse rate $(p=0.01)$. Several molecular pathways associated with aggressive tumor biology regarding cell cycle, Myc, and mTOR downstream signaling were highly enriched in the high glucose uptake subtype as well as distinct response for immunotherapy. MCT4 inhibition suppressed pancreatic cancer cell lines in vitro and showed a synergetic effect with gemcitabine treatment.

Conclusions: MCT4 was identified from integrative analysis as a potential therapeutic target in resectable pancreatic cancer. The precision strategy for resectable pancreatic cancer should be validated in a clinical setting with a prospective study design. 